\title{
Newtonian Semiclassical Gravity in the Ghirardi-Rimini-Weber Theory with Matter Density Ontology
}

\author{
Maaneli Derakhshani* \\ July 21, 2018 \\ Department of Physics \& Astronomy, Clemson University, \\ Clemson, SC 29634, USA 1
}

\begin{abstract}
We propose a Newtonian semiclassical gravity theory based on the GRW collapse theory with matter density ontology (GRWm), which we term GRWmN. The theory is proposed because, as we show, the standard Newtonian semiclassical gravity theory based on the Schroedinger-Newton equations does not have a consistent Born rule probability interpretation for gravitationally self-interacting particles and implies gravitational cat states for macroscopic mass superpositions. By contrast, we show that GRWmN has a consistent statistical description of gravitationally selfinteracting particles and adequately suppresses the cat states for macroscopic superpositions. Two possible routes to experimentally testing GR$\mathrm{WmN}$ are also considered. We conclude with a discussion of possible variants of GRWmN, what a general relativistic extension would involve, and various objections that might be raised against semiclassical gravity theories like GRWmN.
\end{abstract}

Keywords: GRW, collapse models, Schroedinger-Newton, semiclassical gravity.

\footnotetext{
*Emails: maanelid@yahoo.com and maaneli.derakhshani@unl.edu.

†Tel.: +1 9172570311.

${ }^{1}$ Present address: Department of Physics $\&$ Astronomy, University of Nebraska-Lincoln, Theodore P. Jorgensen Hall, Lincoln, NE 68588, USA.
} 


\section{Introduction}

The problem of how to consistently couple a classical gravitational field to quantized matter was first addressed by Moeller [1] and Rosenfeld [2] in the 1960's, who proposed the semiclassical Einstein equation (also called the "MoellerRosenfeld" equation)

$$
G_{n m}=\frac{8 \pi G}{c^{4}}<\psi\left|\hat{T}_{n m}\right| \psi>
$$

where $<\psi\left|\hat{T}_{n m}\right| \psi>$ is interpreted as the (either second quantized or first quantized) quantum expectation value of the stress-energy-momentum tensor operator $\left(\hat{T}_{n m}[\hat{\phi}, g]\right.$ in the second quantized case and $\hat{T}_{n m}(\hat{x}, g)$ in the first quantized case). Motivated from standard quantum mechanics, this is argued [3, 4] to be the only consistent way of incorporating a quantum description into the right hand side of (1) while keeping the left hand side a classical field2. Equation (1) can also be formally derived from the semiclassical approximation of the Wheeler-deWitt equation in canonical quantum gravity, for $\mathrm{N}$ quantum matter fields interacting with the quantized gravitational field as $\mathrm{N} \rightarrow \infty$ [4, 5]; it can also be motivated by various (non-string theoretic) approaches to emergent gravity [6, 7, 8, 9].

It is noteworthy that (1) is the basis for several major results in theoretical astrophysics in the 70's and 80's - Hawking radiation from black holes, the cosmological perturbations generated in cosmic inflation, particle pair production on expanding spacetimes, the creation of naked black hole singularities, traversable wormhole solutions, and warp drive spacetimes, to name a few [10, 11. In the 90's and 2000's, a semiclassical gravity theory known as "stochastic gravity" [5] was proposed as a theoretical bridge between (1) and the as-yet-unknown theory of quantum gravity, and has led to new theoretical predictions for the aforementioned astrophysical phenomena. And most recently, a variant of the semiclassical gravity theory based on (1), known as "gravitational-aether" theory, has been proposed as an observationally testable solution to the old cosmological constant problem [12] and as a novel solution to the endpoint of gravitational collapse in black holes [13]. In addition to these general relativistic results, a nonrelativistic approximation of (1) known as the Schroedinger-Newton (SN) equations has been used by several researchers [3, 14, 15, 16, 17, 18, 19, 20, 21, 22, 23, 24, to predict nonlinear semiclassical gravitational effects that could be observable in future experiments with macro-molecular interferometry [3, 14, 18, 19, 21, 24, 25, 26] and other macroscopic/mesoscopic quantum systems [20, 23, 24, 27. Thus, research on semiclassical gravity theories based on (1) is a highly active area, with potentially

\footnotetext{
${ }^{2}$ The other possible approaches on might try are 1) to equate the left hand side with the stress-energy tensor operator, and 2) to make an eigenvalue equation out of the Einstein equation. The first possibility doesn't make physical or mathematical sense because one equates a c-number with an operator, while the second possibility fails because the components of the stress-energy tensor don't commute and cannot be simultaneously diagonalized.
} 
significant implications for theoretical astrophysics, cosmology, and quantum gravity phenomenology.

However, in spite of all these theoretical motivations and applications, the formulation of semiclassical gravity based on (1) has serious difficulties in its foundation, among them being the lack of a consistent Born rule probability interpretation for gravitationally self-interacting nonrelativistic particles [24, and the well-known gravitational 'cat-state' solutions [4, 10, 15, 17] which are known to be experimentally ruled-out [28]. Both problems have been previously discussed in the context of the SN equations [4, 10, 15, 17, 24], and the primary aim of this paper is to propose a modification of the SN equations in which the collapse dynamics of Ghirardi-Rimini-Weber (GRW) [29, 30] is used to give a consistent statistical description of gravitationally self-interacting particles, and to adequately suppress the cat state solutions for macroscopic superpositions.

We begin our paper by reviewing the arguments showing inconsistency with the Born rule interpretation, and suggest what the correct physical interpretation of the SN equations should be. We then review the existence of macroscopic gravitational cat-state solutions by way of a simple example and show that such solutions, coupled with the correct physical interpretation of the SN equations, imply that the SN equations do not correctly describe the semiclassical gravitational field for macroscopic matter distributions. Next we develop the GRW collapse modification of the SN equations, showing how it leads to a consistent statistical description of gravitationally self-interacting particles and adequately suppresses cat states for macroscopic superpositions. We then discuss experimental possibilities for testing our GRW modification of the SN equations, by re-examining two physical scenarios within which the $\mathrm{SN}$ equations have been recently suggested to make new predictions within experimentally testable reach. We conclude the paper with a discussion of possible variants of our proposed GRW modification, a discussion of what would be involved in a general relativistic extension, and a discussion of possible objections that might be raised against semiclassical gravity theories more generally.

\section{Inconsistency with the Born rule interpretation}

To illustrate the inconsistency with the Born rule interpretation for gravitationally self-interacting particles, we first take the Newtonian limit of (1), making the simplifying assumption that $|\psi\rangle$ is a first-quantized wavefunction and $\hat{T}_{n m}=\hat{T}_{n m}(\hat{x}, g)$ so that (1) specifically corresponds to the EinsteinKlein-Gordon system [31, 32]. With the approximations $g_{n m}=\eta_{n m}+h_{n m}$, $\left|T^{i j}\right| / T^{00}<<1$, and $v<<c$, it can readily be shown 31, 32, that (1) reduces to the semiclassical Newton-Poisson equation,

$$
\nabla^{2} V(x, t)=4 \pi G m|\psi(x, t)|^{2},
$$

with solution

$$
V(x, t)=-G \int \frac{m\left|\psi\left(x^{\prime}, t\right)\right|^{2}}{\left|x-x^{\prime}\right|} d^{3} x^{\prime},
$$


and $\psi$ satisfying the nonlinear integro-differential Schroedinger equation,

$$
i \hbar \partial_{t} \psi(x, t)=-\frac{\hbar^{2}}{2 m} \nabla^{2} \psi(x, t)+m V(x, t) \psi(x, t) .
$$

The N-body generalizations 3 (ignoring the interaction potential term for simplicity) are as follows:

$$
\nabla^{2} V(x, t)=4 \pi G \int d x_{1}^{\prime} \ldots d x_{N}^{\prime}\left|\psi\left(x_{1}^{\prime} \ldots x_{N}^{\prime}, t\right)\right|^{2} \sum_{i=1}^{N} m_{i} \delta^{3}\left(x-x_{i}^{\prime}\right),
$$

and

$$
i \hbar \partial_{t} \psi\left(x_{1} \ldots x_{N}, t\right)=-\sum_{i=1}^{N} \frac{\hbar^{2}}{2 m_{i}} \nabla_{i}^{2} \psi\left(x_{1} \ldots x_{N}, t\right)+\sum_{i=1}^{N} m_{i} V\left(x_{i}, t\right) \psi\left(x_{1} \ldots x_{N}, t\right)
$$

with the solution to (4) given by

$$
V\left(x_{i}, t\right)=-G \sum_{j=1}^{N} \int \frac{m_{j}\left|\psi\left(x_{1}^{\prime} \ldots x_{N}^{\prime}, t\right)\right|^{2}}{\left|x_{i}-x_{j}^{\prime}\right|} d x_{1}^{\prime} \ldots d x_{N}^{\prime}
$$

The coupled equations defined by (2)-(4) or (5)-(6) are the single and many particle SN equations respectively [4, 17. Following Adler 24, we now make the independent particle approximation of (5)-(7) by introducing the ansatz,

$$
\psi\left(x_{1} \ldots x_{N}, t\right)=\prod_{r=1}^{N} \psi_{r}\left(x_{r}, t\right)
$$

with each single particle $\psi_{r}$ normalized through

$$
\int d^{3} x_{r}\left|\psi_{r}\left(x_{r}, t\right)\right|^{2}=1
$$

Using (8), Adler shows in a straightforward derivation that (6)-(7) take the form

$$
i \hbar \partial_{t} \psi_{s}\left(x_{s}, t\right)=-\frac{\hbar^{2}}{2 m_{s}} \nabla_{s}^{2} \psi_{s}\left(x_{s}, t\right)+U\left(x_{s}, t\right) \psi_{s}\left(x_{s}, t\right),
$$

where

\footnotetext{
${ }^{3}$ It should be noted that our assumption that the SN equations have a valid N-body generalization presumes a particular approach to (1); namely, the approach of Moeller and Rosenfeld, Kibble and Randjbar-Daemi [33, and Boughn [34, who consider (1) as a fundamental description of gravity for a single particle or field as well as for many particles or fields. By contrast, in canonical quantum gravity, (1) is valid only as a single-body mean-field equation, when one has $\mathrm{N}$ matter fields or particles interacting with the quantized gravitational field as $\mathrm{N} \rightarrow \infty$ [4]. Nevertheless, this difference will not affect the validity of our subsequent analyses, which will apply to both the single-body and N-body versions of the SN equations.
} 


$$
U\left(x_{s}, t\right)=-G \sum_{u=1}^{N} \int d^{3} x_{u}^{\prime} \frac{m_{s} m_{u}\left|\psi_{u}\left(x_{u}^{\prime}, t\right)\right|^{2}}{\left|x_{s}-x_{u}^{\prime}\right|} .
$$

Note that although (10)-(11) has the same structure as the time-dependent single particle Schroedinger equation that one gets by treating the Newtonian inter-particle potential in the Hartree approximation, it differs in that, for $u=s$, it includes a self-interaction term of the form

$$
-G \int d^{3} x_{s}^{\prime} \frac{m_{s}^{2}\left|\psi_{s}\left(x_{s}^{\prime}, t\right)\right|^{2}}{\left|x_{s}-x_{s}^{\prime}\right|}
$$

To see that such a term does not have a consistent Born rule interpretation, we should first consider a term with $u \neq s$,

$$
-G \int d^{3} x_{u}^{\prime} \frac{m_{u} m_{s}\left|\psi_{u}\left(x_{u}^{\prime}, t\right)\right|^{2}}{\left|x_{s}-x_{u}^{\prime}\right|} .
$$

According to the Born rule interpretation, this would say that the gravitational potential felt by particle $s$ at coordinate $x_{s}$, as a result of the presence of particle $u$ at $x_{u}^{\prime}$, is the Newtonian potential $-G m_{u} m_{s} /\left|x_{s}-x_{u}^{\prime}\right|$ weighted by the probability $\left|\psi_{u}\left(x_{u}^{\prime}, t\right)\right|^{2}$ of finding the particle $u$ at $x_{u}^{\prime}$. However, as Adler notes, this interpretation does not apply to the case of $u=s$, since when particle $s$ is at $x_{s}$, the probability of simultaneously finding it at $x_{s}^{\prime}$ is clearly zero. He also shows this in terms of projection operators - for the case $u \neq s$, we have that $P_{u}\left(x_{u}^{\prime}\right) P_{s}\left(x_{s}\right)$ gives a nonzero projector for finding particle $s$ at $x_{s}$ and particle $u$ at $x_{u}^{\prime}$. But for $u=s$, we have that $P_{s}\left(x_{s}^{\prime}\right) P_{s}\left(x_{s}\right)=0$ for all $x_{s}^{\prime} \neq x_{s}$. So the SN equations do not have a consistent Born rule interpretation of the gravitational self-interaction term for individual particles.

As further support for this conclusion, it is noteworthy that van Wezel and van den Brink [35] also concluded that the SN equations cannot recover the Born rule for self-gravitating particles, by studying the SN time-evolution of a two-state mass superposition. Although they claim to be able to recover the Born rule for the two-state superpositon by making the gravitational potential complex-valued, they found that this approach does not work for superpositions of more than two states.

In spite of these arguments, it is still claimed by some researchers [3, 14] that the standard Born rule interpretation remains consistent for the SN equations because $|\psi|^{2}$ still satisfies the familiar continuity equation,

$$
\frac{\partial|\psi|^{2}}{\partial t}=\nabla \cdot\left[\frac{i \hbar}{2 m}\left(\psi^{*} \overleftrightarrow{\nabla} \psi\right)\right]
$$

Adler's arguments are clearly correct, so how do we reconcile this with (14)? It of course does not logically follow that if a density such as $|\psi|^{2}$ satisfies a continuity equation like (14) then it must necessarily be a probability density. If we interpret $m|\psi|^{2}$ as a real physical mass-matter density field in space-time, as opposed to a mean mass density associated with an ensemble of particles, 
then it can certainly satisfy (14) for all times without having a probability interpretation. In this case, we would just interpret (14) as a statement of local mass conservation for a massive 'fluid' of density $m|\psi|^{2}$. Note that this physical interpretation does not suffer from any inconsistency problem when applied to gravitationally interacting and self-interacting particles. For the $u \neq s$ case, we would say that (13) describes the gravitational potential felt by 'particle' $s$ at coordinate $x_{s}$ from the matter density source $m_{u}\left|\psi_{u}\left(x_{u}^{\prime}, t\right)\right|^{2}$ for 'particle' $u$. And for the $u=s$ case in (12), we would just interpret this as the gravitational self-potential felt by the mass-matter density field $m_{s}\left|\psi_{s}\left(x_{s}^{\prime}, t\right)\right|^{2}$ from the point $x^{\prime}{ }_{s}$ to the point $x_{s}$ within the density

We will therefore claim that the correct physical interpretation of the SN equations is that they describe a world in which the wavefunction in configuration space drives the dynamical evolution of a real physical matter density field (or a set of $\mathrm{N}$ matter density fields in the N-particle case) in 3-space, the evolving matter density field(s) sources an evolving classical gravitational potential in 3-space, and this gravitational potential couples back to the wavefunction, thereby altering the dynamical evolution of the matter density field (the socalled gravitational 'back-reaction'). No statistical interpretation enters here. Of course, this also means that the SN equations, correctly interpreted, have no means of giving a consistent statistical description of the semiclassical gravitational effects they predict, making them hard to take seriously as the basis for an empirically viable semiclassical gravity theory.

As a result of reaching the same conclusion about the empirical viability of the SN equations, Adler poses the following questions (which he does not attempt to answer) [24]. Do the problems that we have encountered (inconsistency with the Born rule interpretation) indicate that a semiclassical approach to gravitation is inconsistent, and hence that gravity must be quantized? Or do they only indicate that a modification of the Moeller-Rosenfeld and SN approach should be sought, which will make possible a consistent semiclassical theory of gravitational effects? As we will later see, a modification of the SN equations is indeed possible that leads to a physically and statistically consistent nonrelativistic semiclassical theory of gravitational effects.

\section{Existence of gravitational cat states}

Unfortunately, in addition to lacking a consistent statistical description of gravitationally self-interacting particles, the correct physical interpretation of the SN equations contains another problem for its empirical viability - the existence of gravitational cat states for macroscopic matter distributions. The SN equations can be shown to admit cat states as follows. Elaborating the example by Ford [10], suppose we have a superposition state,

\footnotetext{
${ }^{4}$ This interpretation is in fact identical to the interpretation of the gravitational selfpotential for a continuous mass density in classical Newtonian gravity theory. The only (albeit nontrivial) difference in the SN case is that the mass density is defined in terms of the mod-square of a wavefunction evolving by the SN equations.
} 


$$
\psi_{\text {cat }}=\frac{1}{\sqrt{2}}\left[\phi_{\text {left }}+\phi_{\text {right }}\right],
$$

where each state in the superposition corresponds to a macroscopic matter distribution in a distinct location (a $1000 \mathrm{~kg}$ mass occupying a volume located on the left or right side of a room). Inserting $\psi_{\text {cat }}$ into (2) then gives

$$
\nabla^{2} V_{\text {cat }}=4 \pi G\left[\frac{m}{2}\left|\phi_{\text {left }}\right|^{2}+\frac{m}{2}\left|\phi_{\text {right }}\right|^{2}\right],
$$

with solution

$$
V_{\text {cat }}=-G \int \frac{\frac{m}{2}\left[\left|\phi_{\text {left }}\right|^{2}+\left|\phi_{\text {right }}\right|^{2}\right]}{\left|x-x^{\prime}\right|} d^{3} x^{\prime} .
$$

In other words, we have a semiclassical gravitational field, $\mathbf{g}_{c a t}=-\nabla V_{c a t}$, which is an average of the fields due to the two distributions separately (in this case, the gravitational field is the sum effect of two $500 \mathrm{~kg}$ masses on opposite sides of the room). By contrast, the simplistic application of the measurement postulates of quantum mechanics would tell us that if we were to measure the gravitational field with an external test particle, $m_{\text {test }}$, the particle would feel a gravitational force from a single $1000 \mathrm{~kg}$ matter density source occupying a single location, and in different locations with equal frequency in multiple measurement trials. As we saw in the previous section, such measurement outcomes are not predicted by anything in the (correctly interpreted) SN equations Moreover, Page and Geilker's torson balance pendulum experiment has already disconfirmed the gravitational field predicted by (17) for macroscopic superpositions [4, 28.

It should be remarked that incorporating the effects of environmental decoherence does not get rid of these cat states (for essentially the same reason that decoherence doesn't solve the quantum measurement problem); all decoherence can do is ensure that $\phi_{\text {left }}(q) \cdot \phi_{\text {right }}(q) \approx 0$ (i.e. $\phi_{\text {left }}$ and $\phi_{\text {right }}$ have disjoint supports in configuration space) for all $q=\left(x_{1}, \ldots, x_{N}\right)$ so that there are no interference terms contributing to the right hand side of (16). It should also be emphasized that the gravitational self-localization effect discovered in numerical simulations by Salzman and Carlip [3], Giulini and Grossardt [21, and Van Meter [19], does not solve the cat states problem either - all the self-localization effect potentially does is ensure that each state in the superposition will localize separate, $500 \mathrm{~kg}$ mass distributions around their respective locations in 3-space.

Thus we see that not only are these cat state solutions inconsistent with experiment, there is no known physical mechanism based on the SN equations alone that can suppress them. To incorporate a mechanism that can requires modifying the SN equations in a non-trivial way, as we will see in the next section.

\section{GRWmN}

Given the inconsistency with the Born rule intepretation and the existence of gravitational cat states, it is clear that semiclassical gravity based on the SN 
equations (or the Moeller-Rosenfeld equation) alone is not empirically viable, nor really consistent with the postulates of standard quantum mechanics. We suggest that one straightforward way to modify the SN equations so as to give a consistent physical and statistical description of gravitationally self-interacting particles while also solving the cat states problem, is to develop a semiclassical gravity theory based on an alternative collapse theory to standard quantum mechanics, namely, the GRW theory [29]; and in particular, the variant of the GRW theory with matter density ontology (GRWm) [30]. We develop a nonrelativistic version of such a theory in this section.

\subsection{Defining equations}

For a single-body system, the "GRWm-Newton" (GRWmN) equations are defined as follows. As in our interpretation of the SN equations, we postulate the existence of a real physical matter density field in space-time,

$$
m(x, t)=m|\psi(x, t)|^{2},
$$

which we use as a source in the Newton-Poisson equation,

$$
\nabla^{2} V(x, t)=4 \pi G m(x, t)
$$

where

$$
V(x, t)=-G \int \frac{m\left(x^{\prime}, t\right)}{\left|x-x^{\prime}\right|} d^{3} x^{\prime} .
$$

This gravitational 'self-potential' couples back to the wavefunction via the SN equation,

$$
i \hbar \partial_{t} \psi(x, t)=-\frac{\hbar^{2}}{2 m} \nabla^{2} \psi(x, t)-G m \int d^{3} x^{\prime} \frac{m\left(x^{\prime}, t\right)}{\left|x-x^{\prime}\right|} \psi(x, t),
$$

but now the wavefunction undergoes discrete and instantaneous intermittent collapses according to the GRW collapse law. That is, the collapse time $T$ occurs randomly with constant rate per system of $N \lambda=\lambda=10^{-16} s^{-1}$, where the post-collapse wavefunction $\psi_{T+}=\lim _{t \searrow T} \psi_{t}$ is obtained from the pre-collapse wavefunction $\psi_{T-}=\lim _{t} \tau_{T} \psi_{t}$ through multiplication by a Gaussian function,

$$
\psi_{T+}(x)=\frac{1}{C} g(x-X)^{1 / 2} \psi_{T-}(x)
$$

where

$$
g(x)=\frac{1}{\left(2 \pi \sigma^{2}\right)^{3 / 2}} e^{-\frac{x^{2}}{2 \sigma^{2}}}
$$

is the 3-D Gaussian function of width $\sigma=10^{-7} \mathrm{~m}$, and

$$
C=C(X)=\left(\int d^{3} x g(x-X)\left|\psi_{T-}(x)\right|^{2}\right)^{1 / 2}
$$


is the normalization factor. The collapse center $X$ is chosen randomly with probability density $\rho(x)=C(x)^{2}$, and the space-time locations of the collapses are given by the ordered pair $\left(X_{k}, T_{k}\right)$. Between collapses, the wavefunction just evolves by (21). The generalization to an N-body system is as follows. We have $\mathrm{N}$ matter density fields in 3-space as given by,

$$
m(x, t)=\int d x_{1}^{\prime} \ldots d x_{N}^{\prime}\left|\psi\left(x_{1}^{\prime}, \ldots, x_{N}^{\prime}, t\right)\right|^{2} \sum_{i=1}^{N} m_{i} \delta^{3}\left(x-x_{i}^{\prime}\right),
$$

which act as the source in the Newton-Poisson equation,

$$
\nabla^{2} V(x, t)=4 \pi G \int d x_{1}^{\prime} \ldots d x_{N}^{\prime}\left|\psi\left(x_{1}^{\prime} \ldots x_{N}^{\prime}, t\right)\right|^{2} \sum_{i=1}^{N} m_{i} \delta^{3}\left(x-x_{i}^{\prime}\right) .
$$

The solution of (26) enters into the N-body SN equation,

$i \hbar \partial_{t} \psi\left(x_{1} \ldots x_{N}, t\right)=-\sum_{i=1}^{N} \frac{\hbar^{2}}{2 m_{i}} \nabla_{i}^{2} \psi\left(x_{1} \ldots x_{N}, t\right)-G \sum_{i, j=1}^{N} \int \frac{m_{i} m_{j}\left(x_{j}^{\prime}, t\right)}{\left|x_{i}-x_{j}^{\prime}\right|} d x_{1}^{\prime} \ldots d x_{N}^{\prime}$

and the solution of (27) undergoes collapse according to

$$
\psi_{T+}\left(x_{1}, \ldots, x_{N}\right)=\frac{1}{C} g\left(x_{i}-X\right)^{1 / 2} \psi_{T-}\left(x_{1}, \ldots, x_{N}\right),
$$

with probability density

$$
\rho(X)=C(X)^{2}=\int d x_{1}^{\prime} \ldots d x_{N}^{\prime} g\left(x_{i}^{\prime}-X\right)\left|\psi_{T-}\left(x_{1}^{\prime}, \ldots, x_{N}^{\prime}\right)\right|^{2},
$$

where $i$ is chosen randomly from $1, \ldots, N$.

The equations of GRWmN for a single body say the following - a wavefunction in 3-space, which evolves by (21) and undergoes the random collapse process in (22), drives the dynamical evolution of a matter density field in 3space via (18). When the wavefunction collapses, it localizes the matter density field around a randomly chosen point in 3 -space, with width of $10^{-7}$ meters, with the probability of the randomly chosen point being largest where the modsquared of the uncollapsed wavefunction is largest, as indicated by (23). This evolving matter density field also sources a gravitational potential in 3-space via (20), and this potential couples back to the wavefunction via (21), which in turn alters the evolution of the matter density field via (20) again.

For N-bodies, the wavefunction lives in configuration space $\mathbb{R}^{3 N}$, evolves by (27), and undergoes the collapse process in (28); this wavefunction drives the dynamical evolution of $\mathrm{N}$ matter density fields in 3-space via (25) so that when the wavefunction collapses, it randomly localizes the matter density fields around randomly chosen (non-overlapping) points in 3-space, each of width $10^{-7}$ meters, and with probability density given by (29). As before, each of 
these matter density fields acts as a source for a gravitational potential in 3space that couples back to the N-body wavefunction via (26)-(27), which in turn alters the evolution of the matter density fields via (25) again.

\subsection{Consistent statistical description of gravitationally self- interacting particles}

In contrast to the SN theory 5 , GRWmN gives a consistent physical and statistical description of gravitationally self-interacting particles. To see this, let us reconsider the example in section 2 .

In GRWmN, $m_{u}\left|\psi_{u}\left(x_{u}^{\prime}, t\right)\right|^{2}$ is interpreted as the real physical matter density field $m_{u}\left(x_{u}^{\prime}, t\right)$ for 'particle 6 , with the probability for the collapse center $X_{u}$ given by the separately defined GRW probability law,

$$
\rho\left(X_{u}\right)=\int d^{3} x_{u}^{\prime} g\left(x_{u}^{\prime}-X_{u}\right)\left|\psi_{T-}\left(x_{u}^{\prime}\right)\right|^{2},
$$

with collapse rate per particle of $\lambda=10^{-16} \frac{1}{s}$. One would then say that the gravitational potential felt by particle $s$ at coordinate $x_{s}$ from the matter density field $m_{u}\left(x_{u}^{\prime}, t\right)$ is

$$
-\int d^{3} x_{u}^{\prime} G \frac{m_{s} m_{u}\left(x_{u}^{\prime}, t\right)}{\left|x_{s}-x_{u}^{\prime}\right|}
$$

and this is true whether the matter density for particle $u$ corresponds to the uncollapsed wavefunction evolving by the SN equations or the collapsed wavefunction defined by (22). Hence, if we now consider the case $u=s$, we can see that GRWmN has no problem handling it - as in our interpretation of the SN equations, we would just interpret the expression,

$$
-G \int d^{3} x_{s}^{\prime} \frac{m_{s} m_{s}\left(x_{s}^{\prime}, t\right)}{\left|x_{s}-x_{s}^{\prime}\right|}
$$

as the gravitational self-potential felt by the matter density $m_{s}\left(x_{s}^{\prime}, t\right)=m_{s}\left|\psi_{s}\left(x_{s}^{\prime}, t\right)\right|^{2}$ from the point $x^{\prime}{ }_{s}$ to the point $x_{s}$ within the density; and this interpretation holds whether the matter density for particle $s$ corresponds to the uncollapsed wavefunction or the collapsed wavefunction. Moreover, it is clear that the probability for the matter density for particle $s$ to collapse to center $X_{s}$ continues to be consistently given by (30) for $u=s$.

\footnotetext{
${ }^{5}$ By the "SN theory", we refer to the (in our view) correct physical interpretation of the SN equations discussed in section 2 .

${ }^{6}$ We use parentheticals to emphasize that, just as in GRWm, there are no particles in the fundamental physical ontology of GRWmN since the matter density is a field distribution on space-time; particle-like states can be said to emerge though when the wavefunction collapses and localizes the matter density to a Gaussian distribution of very narrow but finite width of $10^{-7}$ meters.
} 


\subsection{Suppression of gravitational cat states}

The GRWmN wavefunction, when evolving deterministically by the SN equations, also admits gravitational cat states; but because the GRWmN wavefunction undergoes random collapses according to (22) or (28), which scales with the number of particles, those cat states are not macroscopically observable. (Also, the gravitational field produced by a cat state for a single elementary particle is presumably far too weak to be experimentally measured.) For example, for a massive object composed of Avogadro's number of particles, the collapse rate is $\sim 10^{7} \frac{1}{s}$. So the individual matter fields composing the massive object will be localized around definite points in space frequently enough to give the appearance of a macroscopic matter distribution occupying a particular volume of space.

Returning then to the example of a $1000 \mathrm{~kg}$ mass in the cat state $\psi_{\text {cat }}=$ $\frac{1}{\sqrt{2}}\left[\phi_{\text {left }}+\phi_{\text {right }}\right]$, it is clear that the number of systems needed in practice to compose such a matter distribution would imply an astronomically faster collapse rate; and when such collapses take place via (28), equations (26) and (29) say that the result will be the appearance of a single $1000 \mathrm{~kg}$ mass localized on either the left or right side of the room (assuming the collapse center $X$ for each system takes a binary outcome) with equal frequency. More precisely, whereas the uncollapsed matter density (using for simplicity the single-particle equation),

$$
m_{\text {cat }}(x, t)=m\left|\psi_{\text {cat }}\right|^{2}=\frac{1}{2}\left[m_{\text {left }}(x, t)+m_{\text {right }}(x, t)\right],
$$

produces the gravitational potential,

$$
V_{\text {cat }}=-G \int \frac{\frac{1}{2}\left[m_{\text {left }}\left(x^{\prime}, t\right)+m_{\text {right }}\left(x^{\prime}, t\right)\right]}{\left|x-x^{\prime}\right|} d^{3} x^{\prime},
$$

the GRW collapse gives either the state

$$
\psi_{T+, \text { left }}(x)=\frac{1}{C_{l e f t}} g\left(x-X_{\text {left }}\right)^{1 / 2} \psi_{T-, \text { cat }}(x),
$$

or

$$
\psi_{T+, \text { right }}(x)=\frac{1}{C_{\text {right }}} g\left(x-X_{\text {right }}\right)^{1 / 2} \psi_{T-, \text { cat }}(x),
$$

resulting in either the matter density

$$
m_{\text {left }}(x, t)=m\left|\psi_{T+, \text { left }}(x)\right|^{2},
$$

or

$$
m_{\text {right }}(x, t)=m\left|\psi_{T+, \text { right }}(x)\right|^{2},
$$

and either the gravitational potential 


$$
V_{l e f t}=-G \int \frac{m_{l e f t}\left(x^{\prime}, t\right)}{\left|x-x^{\prime}\right|} d^{3} x^{\prime}
$$

Or

$$
V_{\text {right }}=-G \int \frac{m_{\text {right }}\left(x^{\prime}, t\right)}{\left|x-x^{\prime}\right|} d^{3} x^{\prime} .
$$

The collapse center probability densities are correspondingly given by

$$
P_{\text {left }}\left(X_{\text {left }}\right)=C_{\text {left }}^{2}=\frac{1}{2} \int d^{3} x g\left(x-X_{\text {left }}\right)\left|\psi_{T-, \text { cat }}(x)\right|^{2},
$$

and

$$
P_{\text {right }}\left(X_{\text {right }}\right)=C_{\text {right }}^{2}=\frac{1}{2} \int d^{3} x g\left(x-X_{\text {right }}\right)\left|\psi_{T-, \text { cat }}(x)\right|^{2} .
$$

This ensures that the gravitational field deflecting the test particle in a single trial will look like it is due to only one matter density source at only one of the locations, with the right amount of mass, and with equal location frequency in $\mathrm{N}$ trials. In this way, the gravitational field predicted by GRWmN is consistent with that observed in the Page and Geilker experiment, in contrast to standard semiclassical gravity.

Some comments are in order. First, it is known that the GRW collapse causes a spontaneous increase in the average center-of-mass energy for any system of $\mathrm{N}$ equal-mass particles [29, 36]. So one would expect that the mass-equivalent of this increase in average c.o.m. energy would contribute to the observed gravitational field obtained from (39) or (40). In particular, it would slightly increase the gravitational field strength produced by the collapsed matter density on the left or right side and thus predict a slightly stronger deflection of the test particle in the corresponding direction than would be expected from just the bare mass value $m$. While this is true, it is well-established [29, 36] that the rate of increase in average c.o.m. energy due to the collapses is so tiny that it is not macroscopically detectable with current experimental capabilitie:7, making the contribution to the gravitational field produced by (39) or (40) negligible as well.

Second, readers may notice a close parallel of the cat states problem in semiclassical gravity with the quantum measurement problem in the context of the Schroedinger-cat thought experiment. Indeed, it could be said that the cat states problem is a manifestation of the measurement problem in the context of semiclassical gravity, hence why the SN theory (which is based on the standard quantum mechanics that originates the measurement problem) suffers from it and GRWmN (which is free of the measurement problem by virtue of the GRW

\footnotetext{
${ }^{7}$ The rate of average energy increase is given in Ghirardi et al. [29] by the formula $d \bar{E} / d t=$ $\left(\hbar^{2} \lambda / 4 m \sigma^{2}\right)$. For the GRW values of $\lambda$ and $\sigma$, and using $1000 \mathrm{~kg}$ as the mass, this gives a miniscule value of $\sim 10^{-73}$ Joules per second. It would then take $10^{63}$ years to increase the average mass-energy by only $1 \mathrm{~mJ}$.
} 
collapse mechanism) does not. Note, however, that not all proposed solutions to the measurement problem extend successfully to semiclassical gravity. For example, extending the Many-Worlds interpretations of Everett and Schroedinger 37 to the Moeller-Rosenfeld and SN equations leads to the prediction that both branches of $\psi_{\text {cat }}$ should continue to exist macroscopically and produce a semiclassical gravitational field of the form in (17), which we already noted is ruled out by the Page and Geilker experiment. This suggests that, to the extent that one takes semiclassical gravity theory seriously, it can be used as a testing ground for the robustness of any claimed solution to the measurement problem in standard quantum mechanic: 8 .

\subsection{Experimental prospects}

In addition to the GRW collapse process, the branches of the wavefunction in GRWmN can undergo the gravitational self-localization effect observed in numerical simulations of the SN equations for a free Gaussian wavepacket. In particular, Giulini and Grossardt found that for $m=10^{10} \mathrm{amu}$ and initial width of 0.5 microns, a Gaussian wavepacket will undergo self-localization, reach a minimum width of 0.4 microns in 30,000 seconds, and disperse again thereafter 21. We would argue that GRWmN significantly improves the plausibility of this prediction of the SN equations, considering that GRWmN gives a physically and statistically consistent semiclassical description of gravitational effects and the SN theory does not.

As it has been suggested [21, 25, 26] that molecular interferometry experiments with macromolecule clusters may eventually reach this mass scale, it is natural to ask if GRW collapse might also be observable at this mass scale and perhaps happen 'on top of' the self-localization effect. If we make the generous assumption that in GRWmN a mass of $10^{10} \mathrm{amu}$ corresponds to $10^{10}$ particles of $1 \mathrm{amu}$, this gives an approximate collapse rate of $10^{-6} \frac{1}{s}$, or $10^{6} \mathrm{~s}$ for each collapse. In other words, to have a chance of observing a single GRW collapse event, we would have to maintain the coherence time of the wavepacket for a minimum of about 33 times longer than the timescale it takes the self-localization to reach the minimum width.

It remains to be seen whether technological advancements in molecular interferometry that allow for maintaining coherence times of 30,000 seconds will also allow for maintaining coherence times of $10^{6} s$ or greater. Even so, we note that if self-localization is not observed at the mass scale predicted by the SN equations used in GRWmN, this will be sufficient to falsify GRWmN as a semiclassical theory of gravity. And if self-localization is observed, it would be strong evidence for GRWmN or some dynamical collapse variant of GRWmN9.

\footnotetext{
${ }^{8}$ It is interesting to consider whether solutions to the measurement problem based on nonlocal 'hidden-variables', e.g. the de Broglie-Bohm theory or Nelson's stochastic mechanics, can also solve the cat states problem when extended to semiclassical gravity. This will be examined in a forthcoming paper.

${ }^{9}$ It should also be made clear to what extent experimental confirmation or falsification of the predictions of GRWmN could also be taken as experimental confirmation or falsification of the
} 
It is also worth commenting on Yang et al.'s 20] recent observation that the SN equations predict that a single macroscopic quantum object (modeled by a squeezed Gaussian state) evolving in a harmonic potential has a quantum uncertainty that evolves at a different frequency than the standard quantum mechanical eigenfrequency, and that testing such a prediction is within the capabilities of state-of-the-art optomechanical experiments. Yang et al. compute quantum expectation values of quantum operators and implicitly assume the applicability of the Born rule and quantum projection postulate. But given our observations about the inconsistency of the Born rule as applied to the SN equations, this should raise doubts about the plausibility of the method by which they obtained their prediction. Thus, it would seem important to analyze the physical system they consider in the context of a semiclassical gravity theory that does have a consistent statistical description, e.g. GRWmN. In fact, we would expect GRWmN to give expectation values very close to that found by Yang et al., given that the GRW probability density formula (29), and expectation values of GRW operators computed with it, in general gives values very close to - but not exactly equal to - the Born rule distribution of quantum mechanics and expectation values of quantum operators computed with it [29, 40]. If correct, this would give us much greater confidence in Yang et al.'s prediction of a frequency difference, and it would be yet another route to experimentally testing GRWmN. Perhaps an even more feasible prediction to test than either the self-localization effect or the GRW collapse effect.

\section{Discussion}

To summarize, we reviewed the problem of the inconsistency of the SN equations with the Born rule interpretation and the problem of gravitational cat states. We then presented a GRW modification of Newtonian semiclassical gravity, called GRWmN, to solve these problems. In this way, we showed that one can have a nonrelativistic semiclassical gravity theory related to the Moeller-Rosenfeld equation that's empirically viable in its physical and statistical description of

GRW approach to quantum theory more generally. While self-localization not being observed at the predicted mass scale would falsify GRWmN, it would not be sufficient to falsify the GRW approach more generally - if gravity is fundamentally quantized, it is possible that the correct description of quantum gravity is not the Wheeler-deWitt equation of canonical quantum gravity (from which the SN equations are obtained as the semiclassical approximation), but a different approach that has a semiclassical approximation that does not give the SN equations (string theory would be one example); or, if gravity is fundamentally emergent, it is possible that the correct approach to emergent gravity is something different than the approaches which suggest the semiclassical Einstein equation as the emergent description of gravity (the induced-gravity approach of Sakharov 38 would be such an example). It is then logically possible that the GRW theory could work in these alternative approaches. On the other hand, the observation of self-localization would, as we said, be strong, indirect evidence for the existence of the GRW collapse dynamics (or a similar collapse dynamics such as the CSL dynamics [39]), and thus GRWmN. And if, on top of the self-localization effect, the GRW collapse effect is observed directly, or if statistical consequences of the GRW collapse process are confirmed directly, this would be direct confirmation of GRWmN and the GRW dynamics it employs. 
gravitationally self-interacting particles and macroscopic mass superpositions. In addition, we indicated how GRWmN could in principle be experimentally testable with future molecular interferometry experiments, and how it can be used to revise the predictions of a recently proposed macroscopic test of Newtonian semiclassical gravity based on the SN equations.

To the extent that GRWmN is based on a version of GRW theory with a 'primitive ontology 10 (GRWm), one could ask if other GRW theories with primitive ontologies could be extended to semiclassical gravity. Along with GRWm, perhaps the best known GRW theory with a primitive ontology is GRWf [30, where $\mathrm{f}$ stands for the 'flash' ontology, i.e. an ontology in which matter is represented by the space-time locations of the collapsed wavefunction, or the set $F=\left\{\left(X_{1}, T_{1}\right), \ldots,\left(X_{k}, T_{k}\right), \ldots\right\}$. Obviously the flashes can't, on their own, be used as a source for a semiclassical gravitational field, as they are just space-time locations. One could perhaps modify GRWf so that at each flash a point mass is spontaneously produced, which then acts as a source for a gravitational potentia 11. However, such a theory would entail gross violation of mass conservation every time a flash is produced, making it seem rather contrived, even though logically possible. A more straightforward and natural way we can see to retain the flashes in GRWf and also extend it to a Newtonian theory of semiclassical gravity is to add the presence of a matter density field in space-time which, upon collapse, is localized around the flashes. But this is essentially what happens in GRWm, to the extent that the GRW collapse localizes the matter density field around the space-time locations of the collapsed wavefunction. It would then seem artificial and contrived to continue to insist that the flashes constitute the primitive ontology in this approach. So the more natural GRW approach to semiclassical gravity seems to suggest the matter density ontology as the more natural one to describe the physical world, even for cases when gravitational self-interaction effects can be neglected.

Concerning the collapse mechanism, although we based our formulation of semiclassical gravity on the discrete and instantaneous GRW collapse, it seems entirely possible to also use a continuous and non-instantaneous collapse mechanism such as CSL (Continuous Spontaneous Localization) [39], together with a matter density field ontology (see for example [22]). We used the GRW collapse mechanism because it is mathematically the simplest dynamical collapse process for formulating a Newtonian semiclassical gravity model, and because it is already used in GRWm, a theory which is known to match the predictions of standard nonrelativistic quantum mechanics for all current experiments while also having a primitive ontology that can be naturally extended to semiclassical gravity. (Note that Weinberg's recently proposed collapse model 41] contains the GRW collapse process as a special case, and could certainly be used as well.) On the other hand, using the CSL mechanism would have the advantage that it would be testable with molecular interferometry at much lower mass scales (between $10^{6}$ and $10^{8} \mathrm{amu}$, according to Nimmrichter et al. [42]) than GRWmN,

\footnotetext{
${ }^{10}$ Primitive ontology is defined in 30 as just the "variables describing the distribution of matter in 4-dimensional space-time".

${ }^{11}$ This possibility was suggested by T. Norsen [personal communication].
} 
given that the collapse rate in CSL scales quadratically with the total mass $m$ of a system.

Although we restricted our theory to Newtonian semiclassical gravity, it certainly seems possible to extend it to the semiclassical Einstein equation for both first and second quantized wavefunctions. As we will show in a forthcoming paper, what needs to change for this extension are 1) the collapse law and the matter density field definition - rather than using the nonrelativistic GRW collapse law, we would instead use the relativistic GRW collapse law developed by Tumulka in [43, and the relativistic stress-energy-momentum tensor field proposed by Bedingham et al. in [44; 2) for first quantized wavefunctions, the evolution equations should be the first quantized Klein-Gordon or Dirac equation coupled to the semiclassical Einstein equation [31, while for second quantized wavefunctions one should use the functional Schroedinger or functional Dirac equation coupled to the semiclassical Einstein equation 45. It should be noted however that such a theory will have an inconsistency that also plagues standard semiclassical gravity - the covariant divergence of the right hand side of the semiclassical Einstein equation is nonzero upon wavefunction collapse, while the covariant divergence of the left hand side is always zero (i.e. the Bianchi identity). As Tumulka has noted [personal communication], this inconsistency may mean that the semiclassical Einstein equation with a wavefunction undergoing the GRW evolution does not possess any solutions. This is a question that needs further research 12 , in which case, a GRW version of semiclassical gravity would seem to fare no better here at the moment than standard semiclassical gravity.

A statistical inconsistency in semiclassical gravity theories such as the SN equations has also been claimed by Salcedo [47, and which at first glance might seem to apply to GRWmN and its possible general relativistic extension. Apart from the fact that Salcedo assumes the applicability of the Born rule interpretation and quantum project postulate, which as we saw have no consistent application to the SN equations, Barcelo et al. 48, have noted that one can interpret the violation of Salcedo's statistical consistency criterion as an indication that semiclassical gravity theories need to be complemented with the selection of a natural basis in Hilbert space - a notion that seems compatible with the notion of a "pointer basis" in quantum decoherence theory (which can readily be incorporated with the GRW formalism [49]), as well as the fact that the GRW collapse mechanism selects out the pointer basis as the universally preferred basis [29, 49].

One might also be concerned with Gisin's proof [50, that any nonlinear deterministic wave equation, such as the $\mathrm{SN}$ equations or the semiclassical Einstein equation, would allow for superluminal signaling. However, several researchers 51, 52, 53, 14 have identified loopholes in Gisin's proof that arguably would invalidate the application of the proof to the SN equations. Moreover, there is a more direct objection to the claim that Gisin's proof applies to the SN

\footnotetext{
${ }^{12}$ Wald 46 has developed a prescription for measurement in standard semiclassical gravity that can be given a collapse interpretation and also satisfies $\left.\left\langle T_{n m}\right\rangle\right\rangle_{m}=0$, but it is not clear to us if this prescription can be extended to a GRW theory.
} 
equations, let alone GRWmN - the proof assumes the validity of the Born rule and quantum projection postulate, which we have seen are inconsistent with the SN equations, and of course are not part of the GRWmN theory. One might then ask whether the GRW collapse process allows for superluminal signaling. Fortunately, Gisin also showed in [50] that a nonlinear stochastic collapse modification of quantum mechanics equivalent to the GRW theory does not suffer from the problem of superluminal signaling. Hence GRWmN, insofar as it uses the GRW collapse process, will not allow for superluminal signaling either.

Along with these (real and alleged) inconsistencies, a general relativistic extension of GRWmN would also inherit the stability problem of standard semiclassical gravity (i.e. the fact that the semiclassical Einstein equation is a fourth-order system means that some solutions have runaway behavior), and the formally divergent expectation value of $T_{n m}$ [10]. But these latter problems seem to be manageable - Ford [10] has noted for standard semiclassical gravity that there exist adequate renormalization procedures for $\left\langle T_{n m}\right\rangle$, and there exist reasonable proposals for solving the stability problem by either reformulating the semiclassical Einstein equation as an integro-differential equation to eliminate the runaway solutions or by regarding the semiclassical gravity theory as valid only for spacetimes that pass a certain stability criterion.

In light of all these difficulties that seem to arise in attempting to formulate a consistent theory of semiclassical gravity, we would like to anticipate a potential critic who might ask why one should care about doing so. Especially since many physicists ultimately want a full quantum theory of gravity which presumably won't have the consistency problems of semiclassical gravity theory.

First, as mentioned in the introduction, one of the major approaches to quantum gravity, canonical quantum gravity, has the semiclassical Einstein equation as a prediction of its semiclassical approximation [4, 5]. So the semiclassical limit of canonical quantum gravity - on which some of the calculational results of canonical quantum gravity are based [4 - inherits all the problems we've discussed as semiclassical gravity theory taken as fundamental. This would seem to suggest that canonical quantum gravity would also benefit from a GRW-type modification 13 .

Second, it has been argued by some researchers that gravity may be an emergent, collective phenomenon, in which case it would be misguided to try and quantize it [6, 7, 8, 9, 38, 54, 55, 34. This view motivates models of emergent gravity mentioned earlier, which have the semiclassical Einstein equation as the emergent description of the coupling between quantum theory and gravity [6. 7. 8. Like with canonical quantum gravity, then, emergent gravity approaches could also benefit from a GRW-type modification.

Third, we reiterate that semiclassical gravity (in its standard formulation), in spite of its difficulties, has been used to derive several key results in theoretical astrophysics - Hawking radiation, cosmological perturbations in cosmic inflation, particle pair production in expanding spacetimes, the creation of naked

\footnotetext{
${ }^{13}$ In this connection, it would be interesting to explore whether a GRW version of canonical quantum gravity with matter density ontology would, in its semiclassical Newtonian approximation, reduce to our GRWmN theory.
} 
black hole singularities, traversable wormhole solutions, warp drive spacetimes, as just a few examples [10, 11. There are also the more recent modifications of semiclassical Einstein gravity mentioned earlier, such as stochastic gravity and gravitational aether-theory, which offer new physical insights into the aforementioned astrophysical phenomena. To the best of our understanding, both stochastic gravity and gravitational aether-theory share all the previously discussed technical difficulties of standard semiclassical gravity, and so would presumably also benefit from a GRW-type modification 14 .

Fourth, unlike the quantum gravitational phenomena predicted by most quantum gravity theories, many of the predictions of semiclassical gravity theories may be empirically testable in the near future. On the observational astrophysics side, the density perturbations in the CMB spectrum predicted by semiclassical gravitational effects in eternal cosmic inflation (5) may soon be tested with the Planck Satellite's mapping of the CMB power spectrum [56. Aslanbeigi et al. 12 have also shown that gravitational aether-theory makes observationally testable predictions for the gravitational constant of radiation vs. nonrelativistic matter, as well as for the intrinsic gravitomagnetic effect. On the experimental condensed matter side, it has been proposed by Weinfurtner et al. [57] that a condensed matter analogue of Hawking radiation may be observed in experiments using superfluids with supersonic flow velocities. It has also been shown by Barcelo et al. 58 that the prediction of pair production on expanding spacetimes could be tested in a BEC that simulates a quantum field evolving on an expanding spacetime. And of course, on the experimental atomic/molecular physics side, we have the prediction discussed earlier of gravitational self-localization from the SN equations, which may be testable in molecular interferometry experiments of the near future, as well as the quantum-uncertainty frequency difference for a macroscopic object in a harmonic potential, which should be testable with state-of-the-art optomechanical experiments. Given the potential testability of the various astrophysical predictions of standard semiclassical gravity, and the problematic physical foundations on which standard semiclassical gravity rests, it also seems worthwhile to re-

\footnotetext{
${ }^{14}$ In regards to stochastic gravity, it should be noted that $\mathrm{Hu}$ and Verdaguer 5 developed it to deal with cat states (or large "stress tensor fluctuations" as they put it). They propose an "Einstein-Langevin" equation, a linear equation for a stochastic metric perturbation $h_{n m}$ that takes into account the fluctuations of the stress tensor and is superposed on top of the metric $g_{n m}$ satisfying the semiclassical Einstein equation. It seems to us, however, that the EinsteinLangevin equation they propose would prima facie still suffer from the cat states problem for macroscopic superpositions since $g_{n m}$ is still a solution of the semiclassical Einstein equation, and since the solution of the Einstein-Langevin equation still depends on $\left\langle\psi\left|\hat{T}_{n m}\right| \psi\right\rangle$. We would therefore expect that a test particle sent to probe the stochastic gravitational field $g_{n m}+h_{n m}$ for a macroscopic superposition of two masses will not get deflected by a definite mass distribution at a definite spacetime location, but rather will feel a stochastically fluctuating gravitational field that will be an average of the fluctuating gravitational fields due to two spatially separate masses. An analysis of this situation in the context of the Newtonian limit of stochastic gravity has yet to be done, to the best of our knowledge. However, we would expect the Newtonian case to give the same conclusion unless the stochasticity of the metric perturbations can induce dynamical collapse in the pointer basis, as is the case with the phenomenological stochastic noise field in CSL [39.
} 
analyze these predictions with a GRW approach to see if the GRW approach gives differing predictions that could be observed in said tests. In this regard, it has recently come to our attention [Struyve, personal communication] that Landau et al. 59 have constructed and applied CSL variants of GRWmN to a scalar quantum field undergoing cosmic inflation, and shown that such models leads to different $\mathrm{CMB}$ power spectrums than the standard, scale-invariant Harrison-Zel'dovich one. They have also shown that such collapse models are currently consistent with data on the CMB from WMAP. It remains to be seen if such deviations are also consistent with data on the CMB from the more recent Planck satellite.

In sum, we believe semiclassical gravity is a very worthwhile approach for trying to consistently incorporate quantum mechanics and gravity. And we believe dynamical collapse versions of semiclassical gravity, such as GRWmN and its possible relativistic extensions, provide a much more promising route to a consistent formulation of semiclassical gravity than does standard quantum mechanics.

\section{Acknowledgments}

I thank Roderich Tumulka and Sheldon Goldstein for clarifying discussions and helpful comments on an earlier draft of this paper. I also thank Bei-Lok Hu for useful comments on semiclassical gravity theories, as well as two anonymous referees, one for suggesting two useful references and another for asking a simple question that resulted in significant improvements to the paper.

\section{References}

[1] C. Moeller. Les Theories Relativistes de la Gravitation. Colloques Internationaux CNRS 91, CNRS 91, Paris, 1962.

[2] L. Rosenfeld. On the quantization of fields. Nucl. Phys., 40:353, 1963.

[3] S. Carlip. Is quantum gravity necessary? Class. Quant. Grav., 25:154010, 2008, http://arxiv.org/abs/0803.3456.

[4] C. Kiefer. Quantum Gravity, third edition. (Oxford University Press, Oxford), 2012.

[5] B. L. Hu and E. Verdaguer. Stochastic gravity: Theory and applications. Living Rev. Relativity, 11:3, 2008, http://arxiv.org/abs/0802.0658.

[6] G. E. Volovik. Novel Superfluids, chapter The Superfluid Universe, pages 570-618. International Series of Monographs on Physics 156, Volume 1, 2013, http://arxiv.org/abs/1004.0597. 
[7] S. Finazzi, S. Liberati, and L. Sindoni. Cosmological constant: a lesson from bose-einstein condensates. Phys. Rev. Lett., 108:071101, 2012, http://arxiv.org/abs/1103.4841.

[8] B. L. Hu. Emergent/quantum gravity: Macro/micro structures of spacetime. J. Phys. Conf. Ser., 174:012015, 2009, http://arxiv.org/abs/0903.0878.

[9] C. Barcelo, M. Visser, and S. Liberati. Einstein gravity as an emergent phenomenon? Int. J. Mod. Phys., D10:799-806, 2001, http://arxiv.org/abs/grqc/0106002.

[10] L. H. Ford. 100 Years of Relativity - Space-time Structure: Einstein and Beyond, chapter Spacetime in Semiclassical Gravity. World Scientific, 2005, http://arxiv.org/abs/grqc/0504096.

[11] N. D. Birrell and P. C. W. Davies. Quantum Fields in Curved Space. Cambridge University Press, 1982.

[12] S. Aslanbeigi, G. Robbers, B. Z. Foster, K. Kohri, and N. Afshordi. Phenomenology of gravitational aether as a solution to the old cosmological constant problem. Phys. Rev. D, 84:103522, 2011, http://arxiv.org/abs/1106.3955.

[13] M. Saravani, N. Afshordi, and R. B. Mann (2012). Empty black holes, firewalls, and the origin of bekenstein-hawking entropy. http://arxiv.org/abs/1212.4176.

[14] P. J. Salzman. Investigation of the time dependent Schroedinger-Newton equation. PhD thesis, University of California - Davis, 2005.

[15] R. Penrose. On gravity's role in quantum state reduction. General Relativity and Gravitation, 28:581-600, 1996.

[16] R. Penrose. Quantum computation, entanglement and state reduction. Phil. Trans. R. Soc. Lond. A, 356:1927-1939, 1998, http://citeseerx.ist.psu.edu/viewdoc/download?doi=10.1.1.84.7047\&rep=rep1\&type=pdf.

[17] L. Diosi. Gravitation and quantum-mechanical localization of macroobjects. Phys. Lett. A, 105:199-202, 1984.

[18] D. Giulini and A. Grossardt. Gravitationally induced inhibitions of dispersion according to a modified schroedinger-newton equation for a homogeneous-sphere potential. Class. Quant. Grav., 30:155018, 2013, http://arxiv.org/abs/1212.5146.

[19] J. R. van Meter. Schroedinger-newton "collapse" of the wave function. Class. Quant. Grav., 28:215013, 2011, http://arxiv.org/abs/1105.1579. 
[20] H. Yang, H. Miao, D-S Lee, B. Helou, and Y. Chen. Macroscopic quantum mechanics in a classical spacetime. Phys. Rev. Lett., 110:170401, 2013, http://arxiv.org/abs/1210.0457.

[21] D. Giulini and A. Grossardt. Gravitationally induced inhibitions of dispersion according to the schroedinger-newton equation. Class. Quantum Grav., 28:195026, 2011, http://arxiv.org/abs/1105.1921.

[22] L. Diosi. Gravity-related wave function collapse: mass density resolution. In Invited talk at Sixth International Workshop DICE2012, 2012, http://arxiv.org/abs/1302.5365.

[23] L. Diosi. Gravity-related wave function collapse: Is superfluid he exceptional? In Invited talk at the international workshop "Horizon of quantum physics: from foundations to quantum enabled technologies"., 2012, http://arxiv.org/abs/1302.5364.

[24] S. L. Adler. Comments on proposed gravitational modifications of schroedinger dynamics and their experimental implications. J. Phys. A., 40:755-764, 2007, http://arxiv.org/abs/quant-ph/0610255.

[25] K. Hornberger, S. Gerlich, P. Haslinger, S. Nimmrichter, and M. Arndt. Colloquium: Quantum interference of clusters and molecules. Rev. Mod. Phys., 84:157, 2012, http://arxiv.org/abs/1109.5937.

[26] P. Asenbaum, S. Kuhn, S. Nimmrichter, U. Sezer, and M. Arndt (2013). Cavity cooling of free silicon nanoparticles in high-vacuum. http://arxiv.org/abs/1306.4617.

[27] W. Marshall, C. Simon, R. Penrose, and D. Bouwmeester. Towards quantum superpositions of a mirror. Phys. Rev. Lett., 91:130401, 2003, http://arxiv.org/abs/quant-ph/0210001.

[28] D. N. Page and C. D. Geilker. Indirect evidence of quantum gravity. Phys. Rev. Lett., 47:979-982, 1981.

[29] G. C. Ghirardi, A. Rimini, and T. Weber. Unified dynamics for microscopic and macroscopic systems. Phys. Rev. D., 34::470-491, 1986.

[30] V. Allori, S. Goldstein, R. Tumulka, and N. Zanghi (2012). Predictions and primitive ontology in quantum foundations: a study of examples. http://arxiv.org/abs/1206.0019.

[31] D. Giulini and A. Grossardt. The schroedinger-newton equation as nonrelativistic limit of self-gravitating klein-gordon and dirac fields. Class. Quant. Grav., 29:215010, 2012, http://arxiv.org/abs/1206.4250.

[32] F. Siddhartha Guzman and L. Arturo Urena-Lopez. Newtonian collapse of scalar field dark matter. Phys. Rev. D, 68:024023, 2003, http://arxiv.org/abs/astro-ph/0303440. 
[33] T.W. Kibble and S. Randjbar-Daemi. Non-linear coupling of quantum theory and classical gravity. J. Phys. A., 13:141, 1980.

[34] S. Boughn. Nonquantum gravity. Found. Phys., 39:331-351, 2009, http://arxiv.org/abs/0809.4218.

[35] J. van Wezel and J. van den Brink. Schroedinger-newton equation as a possible generator of quantum state reduction. Phil. Mag., 88:1659-1671, 2008, http://arxiv.org/abs/0803.4488.

[36] P. M. Pearle and E. Squires. Bound state excitation, nucleon decay experiments, and models of wave function collapse. Phys. Rev. Lett., 73:1-5, 1994, http://cds.cern.ch/record/260011/files/P00021809.pdf.

[37] Valia Allori, Sheldon Goldstein, Roderich Tumulka, and Nino Zanghi. Many-worlds and schroedinger's first quantum theory. 2009, http://arxiv.org/abs/0903.2211.

[38] M. Visser. Sakharov's induced gravity: a modern perspective. Mod. Phys. Lett., A17:977-992, 2002, http://arxiv.org/abs/gr-qc/0204062.

[39] P. Pearle (2012). Collapse miscellany. To be included in a volume honoring the 80th birthday of Yakir Aharanov. http://arxiv.org/abs/1209.5082.

[40] S. Goldstein, R. Tumulka, and N. Zanghi. The quantum formalism and the grw formalism. Journal of Statistical Physics, 149:142-201, 2012, http://arxiv.org/abs/0710.0885.

[41] S. Weinberg. Collapse of the state vector. Phys. Rev. A, 85:062116, 2012, http://arxiv.org/abs/1109.6462.

[42] S. Nimmrichter, K. Hornberger, P. Haslinger, and M. Arndt. Testing spontaneous localization theories with matter-wave interferometry. Phys. Rev. A, 83:043621, 2011, http://arxiv.org/abs/1103.1236.

[43] R. Tumulka. A relativistic version of the ghirardi-rimini-weber theory. $J$. Statist. Phys., 125:821-840, 2006, http://arxiv.org/abs/quant-ph/0406094.

[44] D. Bedingham, D. Duerr, G. Ghirardi, S. Goldstein, R. Tumulka, and N. Zanghi (2012). Matter density and relativistic theories of wave function collapse. http://arxiv.org/abs/1111.1425.

[45] H. C. Reis. Quantum evolution of inhomogeneities in curved space. Int. J. Mod. Phys., A14:1633-1650, 1999, http://arxiv.org/abs/hep-th/0108175.

[46] R. M. Wald. Quantum Field Theory in Curved Spacetime and Black Hole Thermodynamics. Chicago University Press, Chicago., 1994.

[47] L. L. Salcedo. Statistical consistency of quantum-classical hybrids. Phys. Rev. A, 86:022127, 2012, http://arxiv.org/abs/1201.4237. 
[48] C. Barcelo, R. Carballo-Rubio, L. J. Garay, and R. Gomez-Escalante. Hybrid classical-quantum formulations ask for hybrid notions. Phys. Rev. A, 86:042120, 2012, http://arxiv.org/abs/1206.7036.

[49] M. Schlosshauer. Decoherence, the measurement problem, and interpretations of quantum mechanics. Rev. Mod. Phys., 76:1267-1305, 2004, http://arxiv.org/abs/quant-ph/0312059.

[50] N. Gisin. Stochastic quantum dynamics and relativity. Helvetica Physica Acta, 62:363-371, 1989.

[51] J. Polchinski. Weinberg's nonlinear quantum mechanics and the einsteinpodolsky-rosen paradox. Phys. Rev. Lett., 66:397-400, 1991.

[52] T. F. Jordan. Reconstructing a nonlinear dynamical framework for testing quantum mechanics. Annals of Physics, 225:83-113, 1993.

[53] M. Czachor. Nonlinear schroedinger equation and two-level atoms. Phys. Rev. A, 53:1310-1315, 1996, http://arxiv.org/abs/quant-ph/9501007.

[54] B. L. Hu. Gravity and nonequilibrium thermodynamics of classical matter. Int. J. Mod. Phys., D20:697-716, 2011, http://arxiv.org/abs/1010.5837.

[55] T. Jacobson. Thermodynamics of spacetime: The einstein equation of state. Phys. Rev. Lett., 75:1260-1263, 1995, http://arxiv.org/abs/gr-qc/9504004.

[56] S. M. Feeney, M. C. Johnson, D. J. Mortlock, and H. V. Peiris. First observational tests of eternal inflation: analysis methods and wmap 7-year results. Phys. Rev. D., 84:043507, 2011, http://arxiv.org/abs/1012.3667.

[57] S. Weinfurtner, E. W. Tedford, M. C. J. Penrice, W. G. Unruh, and G. A. Lawrence. Measurement of stimulated hawking emission in an analogue system. Phys. Rev. Lett., 106:021302, 2011, http://arxiv.org/abs/1008.1911.

[58] C. Barcelo, S. Liberati, and M. Visser. Probing semiclassical analogue gravity in bose-einstein condensates with widely tunable interactions. Phys. Rev. A, 68:053613, 2003, http://arxiv.org/abs/cond-mat/0307491.

[59] S. J. Landau, C. G. Scoccola, and D. Sudarsky. Cosmological constraints on non-standard inflationary quantum collapse models. Phys. Rev. D., 85:123001, 2012, http://arxiv.org/abs/1112.1830. 